\title{
BOUNDARY CONDITIONS FOR APPROXIMATE DIFFERENTIAL EQUATIONS
}

\author{
A. J. ROBERTS ${ }^{1}$
}

(Received 14 February 1991; revised 9 August 1991)

\begin{abstract}
A large number of mathematical models are expressed as differential equations. Such models are often derived through a slowly-varying approximation under the assumption that the domain of interest is arbitrarily large; however, typical solutions and the physical problem of interest possess finite domains. The issue is: what are the correct boundary conditions to be used at the edge of the domain for such model equations? Centre manifold theory [24] and its generalisations may be used to derive these sorts of approximations, and higher-order refinements, in an appealing and systematic fashion. Furthermore, the centre manifold approach permits the derivation of appropriate initial conditions and forcing for the models $[25,7]$. Here I show how to derive asymptotically-correct boundary conditions for models which are based on the slowly-varying approximation. The dominant terms in the boundary conditions typically agree with those obtained through physical arguments. However, refined models of higher order require subtle corrections to the previously-deduced boundary conditions, and also require the provision of additional boundary conditions to form a complete model.
\end{abstract}

\section{Introduction}

A vitally important role of mathematics is in the description and analysis of models of dynamical processes. Recently, centre manifold theory [3], and its generalisation to invariant manifolds $[20,26]$, has shown how we can rationally deduce "simplified" low-dimensional models from very detailed, highdimensional dynamical prescriptions. This new invariant manifold approach is extremely appealing for a number of reasons; in particular, it enables a systematic refinement of the approximation [23] (sometimes to the extent of

\footnotetext{
'Department of Applied Mathematics, The University of Adelaide, GPO Box 498, Adelaide 5001, South Australia.

(C) Copyright Australian Mathematical Society 1992, Serial-fee code 0334-2700/92
} 
showing convergence $[16,27])$, it provides correct initial conditions for the model given any initial condition for the original dynamical system [25], and it enables us to transform suitably a forcing of the original dynamics into a forcing fit for the model equations [7]. Thus this rational approach leads to complete modelling approximations.

However, many important dynamic models, although of low dimension when compared to the original, are still of infinite dimension-in which case they are expressed as partial differential evolution equations. Some wellknown examples include: the Taylor model

$$
\frac{\partial C}{\partial t}=-\frac{\partial C}{\partial x}+D \frac{\partial^{2} C}{\partial x^{2}}
$$

of shear dispersion $[34,16]$; the Kuramoto-Sivashinsky [15, 31] equation

$$
\frac{\partial u}{\partial t}+u \frac{\partial u}{\partial x}+\frac{\partial^{2} u}{\partial x^{2}}+\frac{1}{\Lambda^{2}} \frac{\partial^{4} u}{\partial x^{4}}=0
$$

which models flame fronts, interfacial shear dynamics, directional solidification and weakly two-dimensional turbulence [1]; the Boussinesq

$$
\begin{array}{r}
\frac{\partial \eta}{\partial t}+h_{0} \frac{\partial u}{\partial x}+\frac{\partial}{\partial x}(\eta u)=0 \\
\frac{\partial u}{\partial t}+g \frac{\partial \eta}{\partial x}+u \frac{\partial u}{\partial x}+\frac{1}{3} g h_{0}^{2} \frac{\partial^{3} \eta}{\partial x^{3}}=0
\end{array}
$$

and Kortweg-deVries

$$
\frac{\partial \eta}{\partial t}+c_{0}\left(1+\frac{3}{2 h_{0}} \eta\right) \frac{\partial \eta}{\partial x}+\gamma \frac{\partial^{3} \eta}{\partial x^{3}}=0
$$

equations for long waves in water [36] and in other media; beam equations governing the flexure of a thin beam [27], and in particular, Rayleigh's equation

$$
\alpha \frac{\partial^{4} \eta}{\partial x^{4}}+\rho \frac{\partial^{2} \eta}{\partial t^{2}}-\beta \frac{\partial^{4} \eta}{\partial x^{2} \partial t^{2}}=0
$$

for the bending [11, page 320] and Love's equation

$$
\rho\left[\frac{\partial^{2} \xi}{\partial t^{2}}-\alpha \frac{\partial^{4} \xi}{\partial x^{2} \partial t^{2}}\right]=E \frac{\partial^{2} \xi}{\partial x^{2}}
$$

for longitudinal waves [11, page 326]; the Ginzburg-Landau equation

$$
\frac{\partial A}{\partial t}=\alpha A-\beta \frac{\partial^{2} A}{\partial x^{2}}+\gamma A|A|^{2}
$$

as might arise in convection [21,29] and which, in a complex form, describes the nonlinear evolution of a dispersive wave-train [36, page 601] when it is 
called the nonlinear Schrödinger equation (for deep-water waves, a fourthorder refinement has been calculated by Dysthe [9] and corrected by Janssen [14]). Invariant manifold theory can place these models, all based on slow variations in space, on a rational basis [24]. However, for these "continuum" models, not only do we have to provide the correct initial conditions and forcing, but we also have to provide appropriate boundary conditions at the extremes of the domain of interest. Using the concept of invariant manifolds to do this rationally for such one-dimensional continuum equations is the subject of this paper.

To date, apart from evading the problem altogether by assuming an infinite domain, most boundary conditions used for these models have fallen into one of three categories. Frequently, researchers studying the dynamics of these models choose to use periodic boundary conditions, $[15,31,1]$ for example. A more common procedure is to allow only boundaries in the original problem which fit neatly into the scheme of the asymptotic approximation, [4, 29] for example. The above two choices are forced by the inability of primitive asymptotic schemes, such as the method of multiple scales, to embrace the presence of typical physical boundaries. ${ }^{2}$ The third category are boundary conditions based on physical principles. Frequently the actual conditions at the ends of the physical domain do not fit the modelling approximations; inhomogeneous boundary conditions being outstanding examples. In these cases, heuristic arguments supply suitable boundary conditions for the model. An example is the boundary conditions to be used with beam theory for the idealisations of free, fixed or pinned ends. The lack of attention paid to the derivation of boundary conditions to be used with these continuum models has meant that some fascinating and important aspects of modelling have been by-passed.

It is occasionally possible to use a spectral transformation in the spatial variable to derive boundary conditions for some idealised conditions at the edge of the physical domain. For example, $I$ have done this for beam theory [27]. However, in practice the physical conditions at the boundary are rarely ideal and the spectral approach cannot be applied. In contrast, the approach which I describe here is quite general.

As an introductory example, I have presented in Section 2 a simple instance of the general argument for the physically important linear problem of shear dispersion in a channel. There I derive boundary conditions for both the second-order Taylor model (1) of dispersion and a third-order refinement.

The essence of a rational derivation of boundary conditions is to under-

\footnotetext{
${ }^{2}$ With difficulty, matched asymptotics can derive some boundary conditions. Cross et al. [8] have done this for two-dimensional convective rolls; however their analysis was necessarily a linear one near the boundary, and yet needed to be of high-order in the interior.
} 
stand a little of the dynamics of the boundary layers which exist near the boundaries of the domain of interest. To use the concepts and techniques developed in dynamical systems theory we must interchange the role of space and time by imagining that the spatial variable $x$ is a "time-like" variable and reducing the true time $t$ to the status of a parameter. Consequently, boundary layers become exponential transients decaying towards the modelled slow variations in the interior. Thus, in Section 3.1, some boundary conditions for a model may be found by appropriately projecting the conditions of the end onto the centre manifold of the model-a technique that for temporal evolution is explained in [25]. Further boundary conditions for the model may be obtained by recognising, Section 3.2, that any rapid exponential transients in the model do not model physical modes of the original dynamics and so additional conditions should be used to eliminate them. That these two principles are generally sufficient to supply the requisite boundary conditions for a low-dimensional model is then established in Section 3.3.

An enormously wide variety of dynamical models is addressed in the general discussion of Section 3. Some further issues of this approach are discussed in Section 4. In particular, the relation between ill-posed (unstable) models and the rational derivation of boundary conditions is examined.

\section{Entry and exit conditions for shear dispersion}

Consider the situation of some material, a tracer, being released into a channel and then dispersing as it is swept downstream. As an example of this process, consider the channel and the physical mechanisms of advection and cross-stream diffusion as shown in Figure 1 (see page 58), where I take the advection velocity to be proportional to

$$
u(y)=\frac{3}{2}\left(1-y^{2}\right) .
$$

The tracer is relatively quickly spread across the channel and thus the usual aspect of interest is the long-term evolution of how the tracer is distributed along the channel-a problem in one spatial dimension. The evolution of the tracer concentration in the long term is then modelled [34] by describing the evolution of the cross-stream average concentration via a partial differential equation in time and the along-channel distance $x$. The question of interest which is addressed in this introductory example is: what are the boundary conditions which are needed at the inlet, $x=0$, and at the outlet, $x=L$, of the channel in order to solve the model partial differential equation?

Smith [33] has already considered this problem and my treatment of it is similar to his. However, there are several important differences: I simplify 


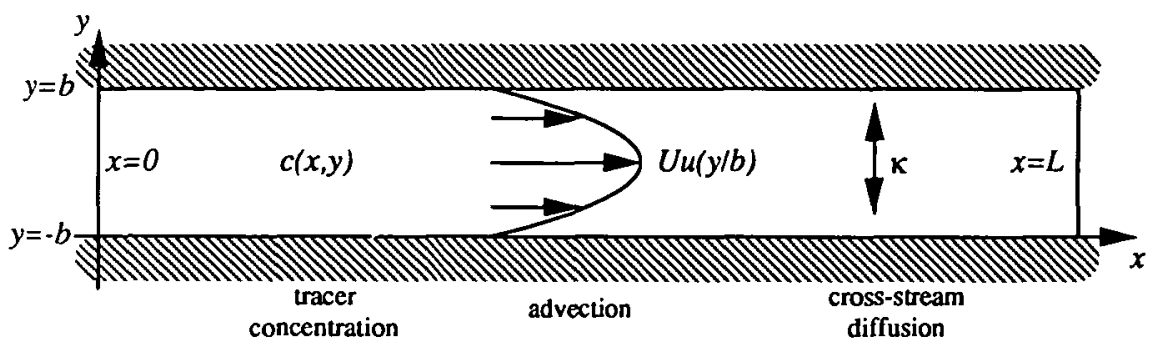

Figure 1. A channel of long length $L$ and the dominant physical mechanisms which lead to shear dispersion of tracer along the channel.

the analysis considerably through performing it to a consistent order of approximation: I extend the analysis to the third-order Taylor model (which models accurately the development of the skewness of the tracer distribution); and I put the analysis in a context which is useful for the later general arguments about the derivation of appropriate boundary conditions.

In order to keep the analysis as simple as possible I non-dimensionalise $y$ with respect to the half channel width $b$, time with respect to a cross-channel diffusion time, $b^{2} / \kappa$, and $x$ with respect to the length, $U b^{2} / \kappa$, which is the downstream advection distance in a cross-channel diffusion time. Thus the governing differential equation expressing conservation of the tracer is

$$
\frac{\partial c}{\partial t}=-u(y) \frac{\partial c}{\partial x}+\frac{\partial^{2} c}{\partial y^{2}} \quad \text { such that } \frac{\partial c}{\partial y}=0 \text { on } y= \pm 1,
$$

in non-dimensional variables. Note that sometimes it will be useful to put results in terms of physical quantities. Also note that in typical geophysical applications, the cross-stream mixing, $\kappa$, is very small and so the time and streamwise-length scales are very large. One consequence of this disparity in the scales is that the effects of streamwise diffusion are very small and hence it has been neglected. However, a short discussion of its influence, when present, is given at the end of Section 3.1.

2.1. The interior: two centre manifold models The Taylor model of the longterm dispersion in a channels relies on the combined action of velocity shear and cross-stream mixing to smooth the tracer distribution. When the tracer distribution is smooth enough, it is nearly constant across the channel and only varies slowly along the channel. Such a smooth tracer distribution need only hold in the interior (with respect to $x$ ) of the channel; that is, it need only hold far away from the effects of the ends at $x=0$ and $x=L$ where conditions may cause "boundary layers" to occur between the ends and the smooth interior distribution. In this subsection, I investigate the dynamics of the tracer in the interior and in the boundary layers. 
Defining $C(x, t)$ to be the cross-stream average concentration, centre manifold theory may be used [16] to show that the long-term evolution of the tracer is given by

$$
\begin{aligned}
c(x, y, t)= & C+\frac{1}{120}\left(-15 y^{4}+30 y^{2}-7\right) \frac{\partial C}{\partial x} \\
& +\frac{1}{201600}\left(675 y^{8}-2940 y^{6}+3570 y^{4}-1020 y^{2}-29\right) \frac{\partial^{2} C}{\partial x^{2}}+\cdots
\end{aligned}
$$

where the mean concentration evolves according to

$$
\frac{\partial C}{\partial t}=-\frac{\partial C}{\partial x}+D \frac{\partial^{2} C}{\partial x^{2}}+e \frac{\partial^{3} C}{\partial x^{3}}+\cdots \quad \text { where } D=\frac{2}{105} \text { and } E=\frac{4}{17325} \text {. }
$$

Abstractly, (10) describes the centre manifold, invariant under the dynamics (9), on which the evolution takes place according to (11). This centre manifold approximation to the dynamics only holds far away from each of the ends, as it is based, in one view, on being able to analyse the integral Fourier transform in $x$ of (9) [24]. Observe the dots at the end of these expressionsthese denote that in principle the expressions extend indefinitely, involving terms with indefinitely many spatial derivatives of $C$. That these infinite sums converge in some sense has been demonstrated by Mercer \& Roberts [16]. The second-order Taylor model (1) is found by just truncating these sums to the first two terms which appear on the right-hand sides. The task is then to solve the second-order version of the partial differential equation (11), whence the details of the corresponding tracer concentration are given by (10). However, what are the appropriate boundary conditions to be used at the ends of the channel, $x=0$ and $x=L$ ? Furthermore, when one may include more and more terms in (10-11) to form a more and more refined model of the dispersion, what are the necessary boundary conditions of the resultant higher and higher order partial differential equation?

These questions first lead us to consider in detail how the tracer concentration makes the transition from whatever the actual end conditions are, to the smooth interior distribution. Physically, we know that the tracer is swept downstream and, in the absence of streamwise diffusion, the conditions at the exit $x=L$ cannot affect the tracer in the channel-the distribution of the tracer is solely determined by the upstream conditions at $x=0$. To see this expressed in the mathematics, I follow Smith [33] and take the Laplace transform of the governing equation (9), assuming for simplicity that initially there is no tracer in the channel, to deduce

$$
\frac{\partial \hat{c}}{\partial x}=\frac{1}{u(y)} \frac{\partial^{2} \hat{c}}{\partial y^{2}}-\frac{1}{u(y)} p \hat{c}
$$


where transformed quantities are denoted by - and the Laplace transform is defined by $\hat{f}(p)=\int_{0}^{\infty} f(t) e^{-p t} d t$. In this form the equation looks like an evolution equation, with $x$ playing the role of a "time-like" variable, which we may integrate from the two ends into the interior of the channel.

Now, the dependence upon the "true time" is represented by the parameter $p$ of the Laplace transform. Provided the applied conditions at the ends of the channel are not varying rapidly in time, then the only significant values of $p$ are small values corresponding to relatively slow variations (in time) in the tracer concentration $c$; thus the second term on the right-hand side of (12) should be thought of as a small perturbation term. Within dynamical systems theory this is best accomplished by imagining $p$ to be an independent variable satisfying the trivial evolution equation

$$
\frac{\partial p}{\partial x}=0
$$

whence the term $p \hat{c} / u(y)$ is interpreted as a small nonlinear term. Linearly (in this new sense) the evolution from the ends into the interior is governed by

$$
\begin{aligned}
& \frac{\partial \hat{c}}{\partial x}=\frac{1}{u(y)} \frac{\partial^{2} \hat{c}}{\partial y^{2}} \quad \text { such that } \frac{\partial \hat{c}}{\partial y}=0 \text { on } y= \pm 1, \\
& \frac{\partial p}{\partial x}=0 .
\end{aligned}
$$

The operator on the right-hand side has a discrete spectrum of $\lambda=0$ (twice), $-\alpha_{1},-\alpha_{2},-\alpha_{3},-\alpha_{4}, \ldots$ where the $\alpha_{n}$ are the eigenvalues of a SturmLiouville problem with the numerically obtained values (via the $N A G$ routine $D 02 K A F) \alpha_{1}=3.4144, \alpha_{2}=12.2535, \alpha_{3}=26.4406, \alpha_{4}=45.9679$, etc.

Thus, in integrating the system (12-13) from the entry to the channel, $x=0$, towards the interior, I apply centre manifold theory [3] and deduce that for all sufficiently small $p$ and for all "initial" conditions, $\hat{c}=\hat{c}_{0}(y)$ at $x=0$, the system evolves exponentially quickly to a smooth two-dimensional centre manifold which is conveniently parameterised by $p$ and $\widehat{C}$ (the crossstream average of $\hat{c}$ )-the exponential approach is roughly like that of the slowest linear transient, namely $\exp \left(-\alpha_{1} x\right)$, and physically this occurs on a length scale of $U b^{2} / \kappa$. The centre manifold and the evolution thereon represents the long-term shear dispersion which takes place in the interior of the channel; that is, it forms an alternative description of the dynamics of the interior low-dimensional model (11). Using techniques described by 
Coullet \& Spiegel [6] and Roberts [23], I find the centre manifold to be

$$
\begin{aligned}
\hat{c}= & \widehat{C}-\frac{1}{120}\left(-15 y^{4}+30 y^{2}-7\right) p \widehat{C} \\
& +\frac{1}{201600}\left(675 y^{8}-2940 y^{6}+3090 y^{4}-60 y^{2}-253\right) p^{2} \widehat{C}+\cdots
\end{aligned}
$$

on which the evolution takes place according to (13) and

$$
\frac{\partial \widehat{C}}{\partial x}=-p \widehat{C}+\frac{2}{105} p^{2} \widehat{C}-\frac{116}{121275} p^{3} \widehat{C}+\cdots
$$

These may also be obtained by reversion of (11) which is then substituted into (10).

The above equations (14-15) model the $x$-evolution in the interior. The question is: what value of $\widehat{C}$ should be used at $x=0$ to integrate (15), in order for (14) to match accurately the exact solution of (12) in the interior? I have previously resolved [25] the general problem of finding the correct initial conditions to use for a low-dimensional model. Applying the methods developed therein (the analysis is very similar to that appearing in Section 6 of [25]) I find the correct initial condition for the $x$-evolution to be $\widehat{C}(0)=\widehat{C}_{0}$ where

$$
\left(1+\frac{2 p}{105}+\frac{29 p^{2}}{40425}+\cdots\right) \widehat{C}_{0}=\overline{u \hat{c}_{0}}+p \overline{u w_{1} \hat{c}_{0}}+p^{2} \overline{u w_{2} \hat{c}_{0}}+\cdots,
$$

in which $\hat{c}_{0}(y)$ is the prescribed distribution of tracer across the entrance to the channel, the overbar denotes a cross-stream average, and where

$$
\begin{aligned}
& w_{1}(y)=\frac{1}{280}\left(35 y^{4}-70 y^{2}+11\right) \\
& w_{2}(y)=\frac{1}{15523200}\left(51975 y^{8}-226380 y^{6}+200970 y^{4}+69300 y^{2}-21881\right) .
\end{aligned}
$$

Physically (16) is reasonable-the dominant terms in the equation are $\widehat{C}_{0}=$ $\overline{u \hat{c}_{0}}$ which just asserts that the tracer flux, $1 \widehat{C}_{0}$, into the channel across $x=0$ for the centre manifold model is equal to that $\overline{u \hat{c}_{0}}$, for the exact system. However, this systematic approach shows that there are corrections to this simple physical principle in the presence of the time dependencies represented by the $p$ factors. The initial condition (16) for the $x$-evolution provides, upon inversion of the Laplace transform, one boundary condition for the original centre manifold evolution equation (11). For example, upon ignoring all terms of order $p^{2}$ or higher and inverting (16), I deduce the boundary condition

$$
C_{0}(t) \approx \frac{1}{D} e^{-t / D} * \overline{u c_{0}(y, t)}+\frac{1}{D}\left[\overline{u w_{1} c_{0}(y, t)}-\frac{1}{D} e^{-t / D} * \overline{u w_{1} c_{0}(y, t)}\right]
$$


for the centre manifold evolution at the entry, where $*$ denotes the convolution $f(t) * g(t)=\int_{0}^{t} f(\tau) g(t-\tau) d \tau$. A feature of interest, as also noted by Smith [33], is that the boundary condition exhibits a memory of earlier conditions at the entry. Due to the convolutions with $\exp (-t / D)$, this memory fades on a time of $D=2 / 105$ times a cross-channel diffusion time-it is only when the actual entry conditions vary extremely slowly in time that the classic, easily understood entry condition, that $C_{0}(t) \approx \overline{u c_{0}(y, t)}$, is recovered.

Similarly, the conditions at the exit $x=L$ also have to be considered rationally from a dynamical viewpoint-physically we know that the conditions at $x=L$ have no upstream influence. The transition from the end condition to the interior is governed by (12); but now integrating from $x=L$ to the interior is in the negative $x$-direction, that is, $-x$ is "time-like". Thus the appropriate linearisation for small $p$ is

$$
\begin{aligned}
-\frac{\partial \hat{c}}{\partial x} & =-\frac{1}{u(y)} \frac{\partial^{2} \hat{c}}{\partial y^{2}} \quad \text { such that } \frac{\partial \hat{c}}{\partial y}=0 \text { on } y= \pm 1, \\
-\frac{\partial p}{\partial x} & =0 .
\end{aligned}
$$

The operator on the right-hand side has a discrete spectrum of (the negative of that for the $x=0$ end) $\lambda=0$ (twice), $+\alpha_{1},+\alpha_{2},+\alpha_{3}, \ldots$. Thus, upon integrating upstream into the channel there are the two modes with zero growth-rate which correspond to the interior's centre manifold, and there are the exponentially growing modes. Since there are no exponentially decaying modes, there are no modes which must be exponentially close to zero in the interior in order to make a bounded transition from the interior to the exit, and so there are no special conditions which the interior solution has to match. Instead, we rely on the evolution far upstream to produce a smoothly varying interior solution right up to the exit.

2.2. The second-order Taylor model Taylor [34] argued that the dispersion of tracer in the channel could be modelled by the structurally stable, onedimensional, advection-diffusion equation (1) which we now recognise as being the leading terms in the asymptotic expression (11). But what are the boundary conditions on the cross-stream average tracer $C(x, t)$ ? Physically, the difficulty lies in the term $D \partial^{2} C / \partial x^{2}$ which introduces an upstream diffusion, and hence an upstream influence, which is not in the posed original system (9). This need not matter significantly as the model equation only applies to tracer concentrations $C(x, t)$ which are sufficiently smooth, in which case the downstream advection dominates. However, this second-order derivative term does introduce possible exponential transients or boundary layers which have no physical counterpart and which should not be allowed. 
The Laplace transform of (1) gives

$$
D \frac{\partial^{2} \widehat{C}}{\partial x^{2}}-\frac{\partial \widehat{C}}{\partial x}-p \widehat{C}=0 .
$$

Again we view this as an evolution equation in the "time-like" variable $x$. The solutions for small $p$ correspond to the slowly-varying solutions on the centre manifold. However, there are two types of solutions $e^{\lambda x}$ for small $p$ : $\lambda_{1}=-p+D p^{2}+\cdots$ which are slowly-varying in $x$ and thus are the desired variations; and the undesirable $\lambda_{2}=1 / D+p-D p^{2}+\cdots$ which gives a rapid and unphysical variation. At the entry to the channel the rapidly growing solution $e^{\lambda_{2} x}$ will not be present as otherwise there will be rapid variations in the interior; thus there is no need to include any condition to attempt to eliminate this solution. However, near the exit the unphysical solution $e^{\lambda_{2} x}$ decays exponentially as $x$ is decreased from $L$ into the interior and so can give rise to a rapid transient; the only way to avoid this is to eliminate this decaying solution by requiring that

$$
\frac{\partial \widehat{C}}{\partial x}=\lambda_{1} \widehat{C}=\left(-p+D p^{2}\right) \widehat{C}+O\left(p^{3}\right) \text {. }
$$

Writing this to the same order of accuracy as

$$
\frac{\partial \widehat{C}}{\partial x} \approx-\frac{p}{1+D p} \widehat{C}
$$

and inverting the Laplace transform, I deduce the approximate exit condition

$$
\frac{\partial C}{\partial x} \approx-e^{-t / D} * \frac{\partial C}{\partial t} \quad \text { at } x=L .
$$

This exit-condition is of a similar form to the equation (7.3) obtained by Smith [33] $]^{3}$; its main feature is the presence of the convolution, which again indicates a memory effect on the time scale of a cross-channel diffusion time. It is only for extremely slow time variations that the right-hand side is very small, to give the exit condition as dominantly $\partial C / \partial x \approx 0$.

To summarise, the second-order Taylor model of shear dispersion, equation (1), should be solved with the entry condition (17), which ensures that the model is accurate after the entry transients have disappeared, and the exit condition (20), which ensures that the exit does not have an upstream influence.

2.3. The third-order model A feature of dispersion in rivers is the persistent skewness of the concentration profile resulting from a point release. However,

\footnotetext{
${ }^{3}$ Smith gives a more complicated expression, but the two are equivalent to second-order accuracy in the slow variations.
} 
the Taylor model can only predict a normal distribution; to predict skewness, a more refined model is needed [5]. Using centre manifold theory this is achieved simply by retaining one more term in the asymptotic expansionsin this subsection, I consider the third-order evolution equation formed from the terms explicitly shown in (11) which describe the evolution on the centre manifold (10).

The Laplace transform of (11) gives

$$
E \frac{\partial^{3} \widehat{C}}{\partial x^{3}}+D \frac{\partial^{2} \widehat{C}}{\partial x^{2}}-\frac{\partial \widehat{C}}{\partial x}-p \widehat{C}=0
$$

which for small $p$ governs the slowly-varying solutions on the centre manifold. However, there are now three types of solutions $e^{\lambda x}$ for small $p$ : the desired slowly-varying solution with $\lambda_{1}=-p+D p^{2}+\cdots$; and two rapidly varying solutions with $\lambda_{2} \approx 36.4212$ and $\lambda_{3} \approx-118.9212$. At the entry to the channel the rapidly growing solution $e^{\lambda_{2} x}$ will not be present, as otherwise there would be rapid variations in the interior; however, the unphysical solution $e^{\lambda_{3} x}$ may be present as a transient-thus we need a condition at the entry to eliminate this component. To obtain this, write (21) as a set of linear first-order differential equations, find the left-eigenvector corresponding to $\lambda_{3}$, and hence deduce

$$
\begin{aligned}
& \lambda_{3}^{2} E \frac{\partial^{2} \widehat{C}}{\partial x^{2}}+\left(\lambda_{3}+p\right) \frac{\partial \widehat{C}}{\partial x}+\lambda_{3} p \widehat{C}=0 \text { at } x=0 \\
& \text { where } \lambda_{3} \approx-118.9212+0.2345 p+0.003481 p^{2} .
\end{aligned}
$$

This is not a simple condition to invert; however, if the time variations are extremely slow, then it may be easily inverted to give the entry condition

$$
-0.02746 \frac{\partial^{2} C}{\partial x^{2}}+\frac{\partial C}{\partial x}=0 \quad \text { at } x=0 .
$$

Conversely, near the exit there will be no $e^{\lambda_{3} x}$ component in the solution, as it would mean that there were large and rapid variations in the interior; however, the unphysical solution $e^{\lambda_{2} x}$ may be present as a transient-thus, as for the second-order model, we need a condition at the exit to eliminate this component. As immediately above we can ensure this by requiring

$$
\begin{aligned}
\lambda_{2}^{2} E \frac{\partial^{2} \widehat{C}}{\partial x^{2}}+\left(\lambda_{2}+p\right) \frac{\partial \widehat{C}}{\partial x}+\lambda_{2} p \widehat{C}=0 \quad \text { at } x=L \\
\text { where } \lambda_{2} \approx 36.4212+0.7655 p-0.01986 p^{2} .
\end{aligned}
$$

If time variations are extremely slow then this may be easily inverted to give

$$
0.008409 \frac{\partial^{2} C}{\partial x^{2}}+\frac{\partial C}{\partial x}=0 \text { at } x=L
$$


To summarise: the third-order model of shear dispersion in a channel, given by (11), should be solved subject to the three boundary conditions, two at the entry and one at the exit, given by the inverse Laplace transforms of (16), (22) and (24). These boundary conditions generally involve memory integrals. For example, the entry condition (16) will of necessity involve a memory effect due to convolutions with the inverse Laplace transform of $\left(1+2 p / 105+29 p^{2} / 40425\right)^{-1}$, namely with

$$
\exp (-385 t / 29) \sin (34.8958 t) \text {. }
$$

These memory effects apparently decay quite rapidly (at least for this specific problem), but still on a time scale of a cross-channel diffusion time. It is most important to realise that these boundary conditions should not be simplified without good reason: (16) is the dominant condition which ensures that the prediction of the model in the interior of the channel will accurately match that of the original system (9); however, it will only do this if the model has no entry transients which is what (22) ensures. In contrast, the exit condition (24) may be cautiously relaxed, thus introducing an unsightly boundary layer at the exit, as there is no exit condition relevant to the interior which would be affected by such a boundary layer.

\section{Rational derivation of boundary conditions}

Consider the wide class of problems which are broadly similar to the shear dispersion problem discussed in the previous section. Similar in the sense that, no matter how complicated the fine details may be, the essential dynamics of long-term importance may be captured by a one-dimensional model.

Suppose that the one dimension of long-term interest is aligned along the $x$-axis with $0 \leq x \leq L$ being the physical domain. Let the unknown dynamical variables in the problem be denoted by $\mathbf{u}(x, t)$ where any dependence upon transverse coordinates (e.g. $y$ in shear dispersion where u denotes $c(y))$ or upon fast-space scales is implicit in the symbol $\mathbf{u}$. Also suppose, for the sake of working with something definite, that the governing equation for the full dynamics, including all the fine detail of no interest, is governed by an autonomous differential equation of the form

$$
\frac{\partial \mathbf{u}}{\partial t}=\mathscr{L}_{0} \mathbf{u}+\mathscr{L}_{1} \frac{\partial \mathbf{u}}{\partial x}+\frac{\partial^{2} \mathbf{u}}{\partial x^{2}}+\mathscr{N}(\mathbf{u}),
$$

where the operators $\mathscr{L}_{n}$ do not contain any dependence upon the longitudinal variable $x$ (they just operate on the uninteresting transverse or fine-scale structure) and where $\mathscr{N}(\mathbf{u})$ are the nonlinear terms. In any particular problem there could be higher-order $x$-derivatives on the right-hand side (or it 
could be only first-order as in (9)); I leave the necessary modifications of the following arguments to those who encounter such problems. Further, suppose that the operator $\mathscr{L}_{0}$ has an eigenspace of importance: either because it has some eigenvalues with zero real-part and the rest with negative real-part (as in shear dispersion where the eigenvalues with zero real-part are precisely zero) in which case (26) possesses an exponentially attractive centre manifold of interesting solutions; or because it has some zero eigenvalues and the rest non-zero (as in beam theory [27]) in which case (26) possesses a sub-centre manifold [30], or "slow" manifold, of solutions varying slowly in $x$ and $t$. For case of exposition, let the interesting solutions be based on eigenvalues which are precisely zero (cases when this requirement is relaxed are discussed in Section 4.2) and call the corresponding invariant manifold, whether it be centre or sub-centre, the slow manifold.

Letting the low-dimensional variable $\mathrm{s}(x, t)$ parameterise locations on the slow manifold, $\mathbf{u}=\mathbf{v}(\mathbf{s})$, the evolution on the manifold forms the rational model [24] and will be governed by an asymptotic evolution equation of the form

$$
\frac{\partial \mathbf{s}}{\partial t}=\mathscr{G}_{0} \mathbf{s}+\mathscr{G}_{1} \frac{\partial \mathbf{s}}{\partial x}+\mathscr{G}_{2} \frac{\partial^{2} \mathbf{s}}{\partial x^{2}}+\mathscr{G}_{3} \frac{\partial^{3} \mathbf{s}}{\partial x^{3}}+\cdots+(\text { nonlinear terms })
$$

Some examples given in the Introduction are the Taylor model of shear dispersion (11), the Kuramoto-Sivashinsky equation, the beam equations, the Korteweg-deVries equation, the Boussinesq equations, and the GinzburgLandau equation. The frequently glossed over difficulty in all of these models is the provision of appropriate boundary conditions, at the extremes of the physical domain, for any given truncation of the sum of $x$-derivatives on the right-hand side of the model. The principles of a rational method to derive the necessary boundary conditions will now be explained.

3.1. Considerations of the original dynamics The method relies on applying some modern dynamical systems theory, not to the time evolution of the system, but to the spatial evolution. This is done through treating the longitudinal coordinate $x$ as a "time-like" variable. Mielke $[17,18,19]$ and Eckmann \& Wayne [10] have also used the space variable $x$ as a "time-like" independent variable in applications of centre manifold theory; but this is the first to also incorporate the true time, albeit as a perturbing influence, and to consider the implications of having a finite domain. The most appealing way to do this is to remove explicit reference to the true time by taking the Laplace transform, $\hat{f}(p)=\int_{0}^{\infty} f(t) e^{-p t} d t$, of the equations and then writing the equations in terms of the "phase-space" variables, here $\hat{\mathbf{u}}$ and $\hat{\mathbf{u}}^{\prime}=\frac{\partial \hat{\mathbf{u}}}{\partial x}$. Taking the Laplace transform of (26) and adjoining an equation to reflect 
that $p$ is a constant gives

$$
\begin{aligned}
& \frac{\partial p}{\partial x}=0, \\
& \frac{\partial \hat{\mathbf{u}}}{\partial x}=\hat{\mathbf{u}}^{\prime}, \\
& \frac{\partial \hat{\mathbf{u}}^{\prime}}{\partial x}=-\mathscr{L}_{0} \hat{\mathbf{u}}-\mathscr{L}_{1} \hat{\mathbf{u}}^{\prime}+p \hat{\mathbf{u}}-\widehat{\mathscr{N}} .
\end{aligned}
$$

This is in the form of an autonomous dynamical system in the variable $\mathbf{U}=$ $\left(p, \hat{\mathbf{u}}, \hat{\mathbf{u}}^{\prime}\right)^{\top}$ with linear terms given by the operator

$$
\mathscr{L}=\left[\begin{array}{ccc}
0 & 0 & 0 \\
0 & 0 & I \\
0 & -\mathscr{L}_{0} & -\mathscr{L}_{1}
\end{array}\right]
$$

and with nonlinear terms $(0,0, p \hat{\mathbf{u}}-\widehat{\mathscr{N}})^{\mathrm{\top}}$.

Equation (28) governs the evolution in the longitudinal variable $x$ in the presence of slow time variations; in particular, it governs the evolution from the ends, $x=0$ and $x=L$, into the interior. Thus the evolution into the interior depends critically upon the spectrum of $\mathscr{L}$. In addition to the one zero eigenvalue due to the adjoined equation $\partial p / \partial x=0$, there will be at least as many zero eigenvalues of $\mathscr{L}$ as there are of $\mathscr{L}_{0}$. For example, if there are no advection terms, $\mathscr{L}_{1} \partial \mathbf{u} / \partial x$, in the original (26) then $\mathscr{L}$ will have one more than twice as many zero eigenvalues as does $\mathscr{L}_{0}$. Thus, there also exists a centre manifold [3] of the spatial evolution (28). Since the slow manifold model in the interior, equation (27), depends directly upon the zero eigenvalues of $\mathscr{L}_{0}$ and their associated properties, the centre manifold of the spatial evolution (28) and its properties are closely tied to those of the model (27)-they are just different descriptions of the same dynamics (recall, in shear dispersion, that (15) is the reversion of (11)). Now, the operator $\mathscr{L}$ will possess some eigenvalues with positive real-part and some eigenvalues with negative real-part; ${ }^{4}$ based on these eigenvalues there exists a corresponding unstable manifold and a corresponding stable manifold respectively $[12, \S 3.2]$. There also exist combination manifolds such as the centre-stable manifold. These manifolds are drawn schematically in Figure 2 (see page 68).

Upon integrating from an end into the interior we presume that the interior solution is slowly-varying and so the trajectory must lie entirely within the centre-stable manifold. If this were not so then the trajectory would become unbounded exponentially quickly, as is illustrated by the two trajectories drawn in Figure 2. Strictly, in a finite-length domain, the trajectory

\footnotetext{
${ }^{4} \mathrm{Here}$ I will ignore the degenerate case when some of the eigenvalues are non-zero pure imaginary numbers.
} 


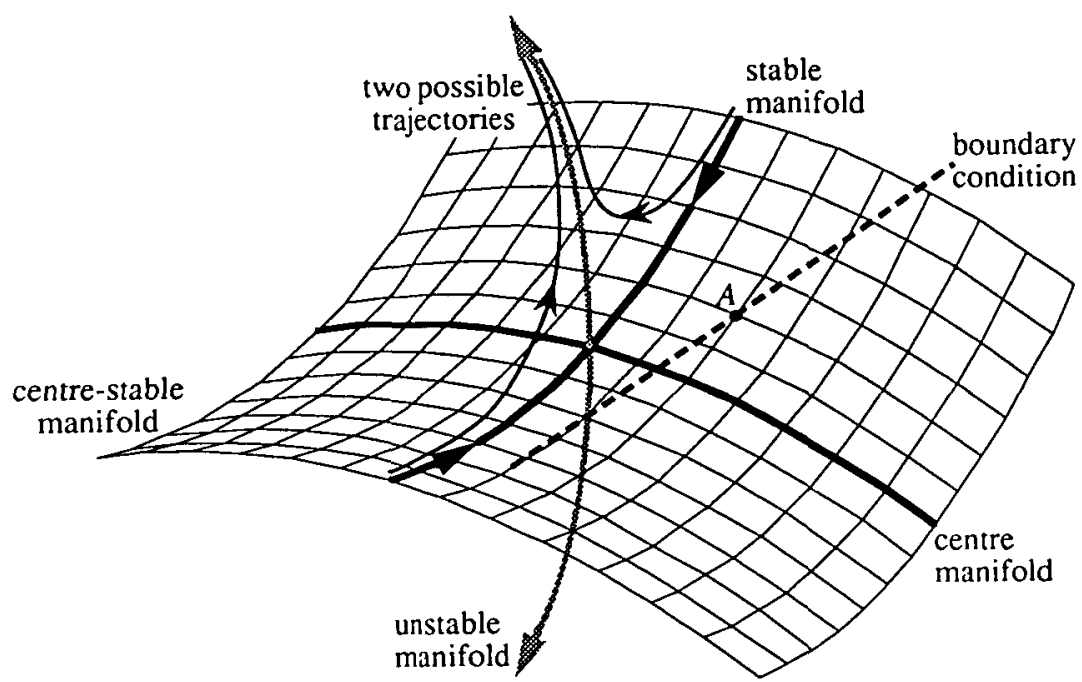

FIGURE 2. A schematic drawing of the centre, stable, unstable and centre-stable manifolds of a dynamical system. Boundary conditions (dashed) restrict how the $x$-evolution starts; but only those points, labelled $A$, on the centre-stable manifold are significant.

need only start exponentially close to the centre-stable manifold so that it stays bounded in the domain of interest $[0, L]$; however, in the arguments which I present, the domain, although finite, is assumed long enough so that being "exponentially close", with a difference $\exp (-\alpha L)$ for some $\alpha$, is effectively the same as being "equal". Now, the boundary conditions of the original problem at the end under consideration, homogeneous or not, linear or nonlinear,

$$
\mathscr{B}\left[t, \mathbf{u}, \mathbf{u}^{\prime}\right]=b(t) \quad \text { or transformed } \quad \widehat{B}\left[\hat{\mathbf{u}}, \hat{\mathbf{u}}^{\prime}\right]=\hat{b}
$$

restricts the $x$-evolution to start somewhere in a specific set of points in the state space; this set of points is drawn schematically as the broken line in Figure 2. The restriction of having to start in the centre-stable manifold is in addition to the boundary conditions of the original problem (26). Thus the possible "initial" conditions for the $x$-evolution into the interior are found by the intersection of the original applied boundary conditions and the centrestable manifold (shown schematically as the point $A$ in the figure). But we are only concerned with the interior, and not at all concerned with any exponential transients. Using techniques to find the appropriate initial condition for a centre manifold model from the initial condition for a high-dimensional dynamical system [25] we may project the set of "initial" conditions for the $x$-evolution (represented by $A$ in the figure) onto appropriate conditions for 
the centre manifold model of (28). But this centre manifold model is intimately linked to the model (27) for the original (26) and so this projection gives a set of boundary conditions for the low-dimensional model (27).

In the example of shear dispersion discussed in the previous section the above considerations were particularly simple. At the entry $x=0$ and integrating in the positive $x$ direction there was no unstable manifold-the entire state space is the centre-stable manifold. Thus the only aspect of the above argument that was needed was to project the given entry concentration of tracer onto the centre manifold, as is achieved by (16) or its approximate version (17). At the exit $x=L$ we have to integrate in the negative $x$ direction and hence there is no stable manifold. Since there is no exit condition to apply, the above argument says that the entire centre manifold is a valid state at the exit. Thus, from these particular considerations, there is no boundary condition for the exit.

It is interesting to note the changes in the argument if the example of dispersion in a channel is modified by including the along-stream diffusion term, $\kappa \partial^{2} c / \partial x^{2}$. Due to the differential scaling of the down-stream and cross-stream coordinates this introduces a perturbing term of $\varepsilon \partial^{2} c / \partial x^{2}$ into (9) where $\varepsilon=(\kappa / U b)^{2}$ is typically very small. Such a second-order term in $x$ effectively doubles the dimensionality of the $x$-evolution in the manner of (28) discussed above. The downstream decaying modes used in Section 2.1 are barely affected by the perturbing term; thus there is no qualitative effect at the entry, there is only a small quantitative perturbation. However, introduced are a whole range of new modes which decay very rapidly upstream, on a length scale of $\varepsilon$. These, together with the neutral modes, form the basis for a centre-stable manifold at the exit. Then the freedom to be anywhere on the centre-stable manifold at $x=L$ is countered by the boundary conditions at the exit (for example, some fixed concentration) so that, after projecting the possibilities onto the centre manifold (10), there are enough degrees of freedom left to cover the entire centre manifold. Hence, even in the presence of this along-stream diffusivity, the exit still provides no boundary condition for the centre manifold model (11).

3.2. Considerations of the model dynamics From analysing the dynamics of the original system (26) we may derive a fixed number of boundary conditions which are appropriate for the model. In the example of shear dispersion, I derived one such boundary condition, namely (16). However, in general the model partial differential equation (27) is any fixed truncation of an asymptotic sum of derivatives of higher and higher order-and, as the order of the approximation is increased, more and more boundary conditions are required to make a well-posed model problem (as seen in Sections 2.2 and 2.3). 
These additional boundary conditions come from an analysis of the spatial transients of the model which, in general, have no physical relevance. Again the most appealing way to proceed is to take the Laplace transform of the model equation (27), in whatever truncation or equivalent asymptotic form is to be used, to give

$$
\mathscr{G}_{0} \hat{\mathbf{s}}+\mathscr{G}_{1} \frac{\partial \hat{\mathbf{s}}}{\partial x}+\cdots+\mathscr{G}_{\kappa} \frac{\partial^{\kappa} \hat{\mathbf{s}}}{\partial x^{\kappa}}=p \hat{\mathbf{s}}-(\text { nonlinear terms }),
$$

where (imagine adjoining $\partial p / \partial x=0$ ) we treat the right-hand side as small nonlinear terms. If the slow manifold (parameterised by $\mathbf{s}$ ) is $l$-dimensional then (29) is equivalent to a coupled system of $\kappa l$ first-order differential equations in the "time-like" variable $x$. Such a dynamical system, as shown schematically in Figure 3, will itself possess an $l$-dimensional slow manifold (which I will take to be the centre manifold ${ }^{5}$ ), together with stable and unstable manifolds, as it is integrated from an end into the interior of the physical domain.

Generalising the arguments of Section 2.3 , the exponentially growing solutions which are characteristic of the unstable manifold are certainly not desirable, as they cause rapidly varying solutions in the interior. However, provided that boundary conditions are applied at the other end which cause the solution to be reasonable, then these rapidly increasing modes will not occur to any significant extent, and hence there is no need to insist on their removal at the end under consideration. Conversely, the exponentially decaying solutions which are characteristic of the stable manifold do not cause any problem in the interior. However, their transient decay is typically unphysical and thus should be removed from the solutions of the model equation. Thus additional boundary conditions for the model come from requiring that it lie in the centre-unstable manifold of (29), as only then will it not have unphysical boundary layers near each end. The fact that the exponentiallygrowing solutions contained in the centre-unstable manifold actually do not occur is due to the boundary conditions at the opposite end of the domainthus with these boundary conditions the solutions to (27) will lie in, or at least exponentially close to, the slow manifold, and the solutions will also lie (almost) in the centre manifold of the asymptotically equivalent (29) (as I have indicated by one of the trajectories in Figure 3).

These considerations lead to the exit condition (20) for the second-order Taylor model of shear dispersion discussed in Section 2.2. Similarly for the more refined third-order model of shear dispersion discussed in Section 2.3,

\footnotetext{
${ }^{5}$ If the slow manifold of the model is not the entire centre manifold, due to some pure imaginary eigenvalues of the operator on the left-hand side of (29), then, as I will argue in Section 4.3, the model equation is not well-posed in that it possesses unphysical temporal behaviour.
} 


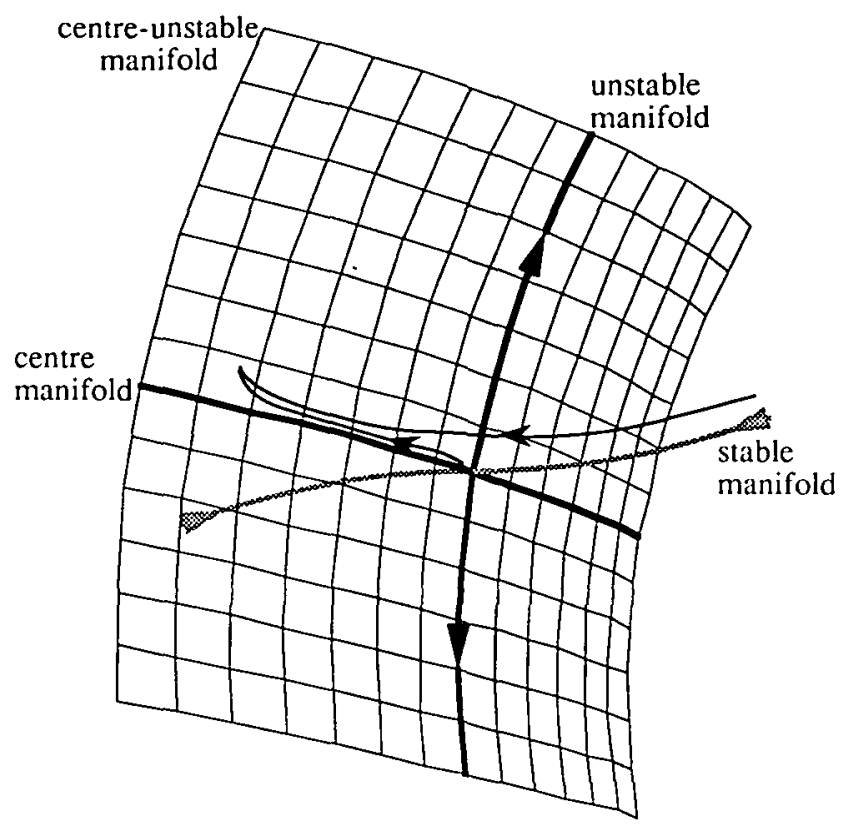

Figure 3. A schematic drawing of the centre, stable, unstable and centre-unstable manifolds of a dynamical system. Two trajectories are drawn; one showing an undesirable transient behaviour off the centre-unstable manifold.

these considerations lead to the different exit condition (24) and to an extra entry condition (22). ${ }^{6}$

3.3. Some dimensional book-keeping It is instructive to comment on the dimensions of the various manifolds mentioned above. In discussing the dimensions, I shall only consider the dimensionality at any fixed $x$, or equivalently as if there were no $x$ dependence. Furthermore, I shall only contemplate the generic case.

Suppose that the original system (26) is $N$-dimensional; that is, $\mathbf{u}(x, t)$ evolves with $x$ and $t$ in a space of $N$ dimensions. This is the dimensionality of the detailed structure, most of which is ignored in the slow manifold model. For example, in shear dispersion the original dimensionality is $N=$ $\infty$ as the cross-stream concentration is a continuous function of $y$. If the highest $x$-derivative in the original system is of order $k$ ( $k=1$ for the shear dispersion equation (9), and $k=2$ for the form (26) discussed earlier in

\footnotetext{
${ }^{6}$ An alternative principle is that any set of boundary conditions is allowed which, when projected as an initial condition, projects to the same conditions as the projection of the boundary conditions for the original system. This principle permits (unphysical) boundary layers in the model, but only those which evolve into the correct interior solutions.
} 
this section) then we would need to have a total of $k N$ boundary conditions specified to make the original a well-posed problem.

Further suppose that the slow manifold, and hence the parameter $\mathbf{s}(x, t)$, is $l$-dimensional. In the shear dispersion example the (slow) centre manifold, parameterised by the cross-stream average concentration, has dimension $l=$ 1. To analyse the spatial transients, the original system is transformed to a set of $k N$ first-order differential equations, analogous to (28) and ignoring the extra dimension introduced by the artifice of adjoining $\partial p / \partial x=0$. Unless $\mathscr{L}_{1}$ in (28) has some special degeneracy, the set of equations will possess a centre manifold which is also of dimension $l$, as exhibited by (14-15) in the shear dispersion example. The evolution, of (28) say, in the positive $x$ direction then must give a stable manifold of some dimension $m$ and an unstable manifold of some dimension $n$ where

$$
l+m+n=k N \text {. }
$$

Since the evolution in the negative $x$ direction, from the end $x=L$ back into the interior, is the reverse of this then the stability of these invariant manifolds is reversed and it has an unstable manifold of dimension $m$ and a stable manifold of dimension $n .^{7}$ Thus the centre-stable manifold at $x=0$ has dimension $m+l$ while that at $x=L$ has dimension $n+l$; a total number of degrees of freedom, for having smooth solutions in the interior, of $m+n+2 l=k N+l$ by (30). Thus the additional $k N$ boundary conditions of the well-posed original problem reduces this to $l$ degrees of freedom to be split between the two ends. At each end, the set of allowed initial conditions has to be projected onto an $l$-dimensional centre manifold $(2 l$ dimensions in all) and so we derive a total of $l$ boundary conditions for the model (27) through the consideration of the dynamics of the full system as explained in Section 3.1.

The analysis of the dimensionality of the dynamics of any truncated version of the model (27) is similar. Suppose the model is truncated to order $\kappa$; that is, the highest spatial derivative term is $\partial^{\kappa} \mathrm{s} / \partial x^{\kappa}$. When considered as a dynamical system in the "time-like" variable $x$, equation (29), the model will have a dimensionality of $\kappa l$. Under the assumption that the operator $\mathscr{G}_{1}$ is generic, the model will have a centre manifold of dimension $l$. Upon considering the evolution in the positive $x$ direction the model will have a stable manifold of dimension $\mu$ and an unstable manifold of dimension $\nu$ where

$$
l+\mu+\nu=\kappa l .
$$

\footnotetext{
${ }^{7}$ It is possible, in the presence of spatial inhomogenities, that this mirror symmetry does not hold. In such a case I would expect that some sort of transition would occur in the interior (maybe a phase transition) which would have to be considered carefully.
} 
Conversely the evolution in the negative $x$ direction will generally have an unstable manifold of dimension $\mu$ and a stable manifold of dimension $\nu$. Thus the centre-unstable manifold of the model at the end $x=0$ will have dimension $l+\nu$, while that at $x=L$ will have dimension $l+\mu$. Insisting that the model's solution lie in these centre-unstable manifolds is thus equivalent to specifying $\mu$ constraints at the end $x=0$ and $\nu$ constraints at $x=L$.

Thus the principle of eliminating rapid boundary layers in the model, as explained in Section 3.2, gives a total of $\mu+\nu$ boundary conditions. These would be used together with the $l$ boundary conditions derived earlier to give a well-posed boundary value problem for the model (27).

\section{Miscellany}

There are a number of issues in this discourse on boundary conditions which are worth commenting upon.

4.1. Shun the Laplace transform Throughout this paper so far I have always resorted to taking the Laplace transform of the governing equations. This was done so that the time variations could be implicitly represented by the parameter $p$, and that slow variations in time correspond to solutions with small $p$. Upon taking the Laplace transform an appeal to the rigorous theory of centre manifolds [3], and other associated manifolds, could easily be done. However, after all the analysis is performed, the only actual role of the parameter $p$ (introduced by the transformation) is to act as a "place-holder" for the operation of differentiating with respect to time. Other than this, the Laplace transformation does nothing but make the execution of the analysis more complicated; for example, by turning multiplicative nonlinearities into awkward convolutions. Thus the whole analysis should be simplified, both in detail and appeal, by not taking the Laplace transform and instead treating $\partial / \partial t$ as if it was the small quantity $p$.

The gain is that there are significantly less complicating details and the analysis deals with the original, physically meaningful quantities, rather than transformed quantities. This is particularly important where the original problem naturally has time variations in it, in which case the Laplace transform would be extremely awkward to deal with; without transformation the analysis is straightforward as can be seen in the analogous case for spatial variations in shear dispersion [16]. The only loss is in the immediate rigour of the analysis. But since the resulting expressions are exactly equivalent, this apparent lack of rigour in the non-transformed analysis merely reflects the current lack of a fully developed theory. I leave the development of such rigorous theory to those with a suitable background in analysis. 
4.2. Models based on an invariant manifold The above arguments for the derivation of the correct boundary conditions have been for the case of a model based on the slow manifold of the dynamics. These models and their derivations are the easiest to understand. However, a rational approach to approximating dynamics should not be limited to this case. For example, a model dominated by oscillating solutions (propagating waves) is obtained from modes which have a growth-rate with zero real-part but non-zero imaginary-part. Furthermore, as I have previously argued [26], effective approximations may be rationally based even when including some modes which are exponentially decaying [22, 32]. These modes form the basis of an invariant manifold. Indeed, this is related to the idea of inertial manifolds [35] which have been proved to exist in some strongly dissipative systems. In essence, which of the modes are kept, to form the basis of the low-dimensional model, depends upon the important time-scale. Modes which decay rapidly are irrelevant, while those which evolve on a time-scale comparable to that of interest, albeit an exponential decay, should be keptthus the dividing time-scale between those modes included in the model and those which are not should be determined by the use to which the model is to be put.

The essence of the above derivation of appropriate boundary conditions will still hold for such invariant manifold approximations. By definition, there are a certain set of modes, those of the relevant slower evolution or the oscillation, which will be modelled accurately by the invariant manifold. Upon transferring the viewpoint from the temporal evolution to the spatial evolution, these same modes should be recognisable both in the original system and in the low-dimensional invariant manifold model. In the above they were recognised by the zero eigenvalues in the spatial evolution equations. Thus we should be able to construct the analogue of the centre-stable manifold of the original system and then project the intersection of this with the actual boundary condition onto the analogue of the centre manifold. These form boundary conditions for the model which ensure that the spatial evolution in the interior matches that of the original system. For the remaining boundary conditions which may be needed for higher-order differential models a distinction should be able to be drawn between the physically accurate modes and the other exponential, physically irrelevant modes. Thus boundary conditions may be found which eliminate unphysical boundary layers near each end. These two types of boundary conditions form the requisite set for the approximation.

4.3. Ill-posed models For high-order differential models, an essential part of the argument given in Section 3.2 is that the centre manifold of the spatial evolution view of the model, (29), is the same as the slow manifold of the spa- 
tial evolution view of the original system, (28). Only then will denying exponential transients give enough boundary conditions for the model. However, this does not always occur-sometimes the centre manifold of the model has modes in addition to the physically relevant ones.

An example of this is seen in the Boussinesq approximation for long waves in water which is given by equations (3-4) [36, page 461] for water of height $y=h_{0}+\eta(x, t)$ above a flat bottom and with average horizontal velocity $u(x, t)$. The Boussinesq approximation is a refinement of the long-wave approximation

$$
\frac{\partial \eta}{\partial t}+h_{0} \frac{\partial u}{\partial x}=0 \text { and } \frac{\partial u}{\partial t}+g \frac{\partial \eta}{\partial x}=0
$$

in that it incorporates the leading-order effects of dispersion, through the $\frac{1}{3} g h_{0}^{2} \frac{\partial^{3} \eta}{\partial x^{3}}$ term, and nonlinear dynamics, though the terms $\frac{\partial}{\partial x}(\eta u)$ and $u \frac{\partial u}{\partial x}$. Its basis is the two modes, waves travelling to the right and waves travelling to the left, which evolve slowly when the waves are of long wavelength (small wavenumber). To derive boundary conditions for the Boussinesq equations (3-4) we should investigate the linearised spatial evolution for modes which evolve slowly in time; that is, substitute a solution proportional to $\exp (p t+\lambda x)$ and, ignoring terms multiplied by $p$ as being small, observe solutions only for $\lambda=0$ (twice) and for $\lambda= \pm i \sqrt{3} / h_{0}$. The two zero eigenvalues correspond to the slow manifold of the long wave approximation. The two pure-imaginary eigenvalues correspond to unphysical modes which we need to eliminate from the solution by imposing appropriate boundary conditions. But, because they are not exponential, it will be difficult for boundary conditions imposed at the ends of the domain to do this.

The linearised temporal evolution shows that these modes are linked to the presence of unphysical instabilities in the Boussinesq equations. Linearising the equations (3-4) and substituting solutions with wavenumber $k$ and growth-rate $\sigma$, that is, proportional to $\exp (\sigma t+i k x)$, we find the relation

$$
\sigma= \pm i \sqrt{g h_{0}} k \sqrt{1-\frac{1}{3} h_{0}^{2} k^{2}} .
$$

For genuinely long waves, small $k$, this gives the dispersion relation

$$
\omega=i \sigma \sim \pm \sqrt{g h_{0}} k \mp \frac{1}{6} \sqrt{g h_{0}^{5}} k^{3}
$$

which is asymptotically correct, having an error $O\left(k^{5}\right)$ when compared to the exact dispersion relation of $\omega= \pm \sqrt{g k \tanh \left(k h_{0}\right)}$. However, the finite 
wavenumber behaviour of the Boussinesq equations is dramatically wrong. For wavenumbers $|k|>h_{0} / \sqrt{3}$ the growth-rate $\sigma$ is no longer pure imaginary, characteristic of the physical waves, but is instead real with one growthrate of the pair positive and the other being negative-for high wavenumbers there is an unphysical instability in the model. In principle, this need not matter; all we need to do to eliminate these temporally-unstable modes is to impose a condition of boundedness on the solution at large time, in place of extra boundary conditions at either end. In practice, such a condition is impractical to implement. Instead the usual preferred path is to change the model equations with the aim of maintaining the asymptotic validity for long waves, while removing the unstable behaviour at finite wavenumber. Boussinesq favoured the system [36, page 462]

$$
\begin{array}{r}
\frac{\partial \eta}{\partial t}+h_{0} \frac{\partial u}{\partial x}+\frac{\partial}{\partial x}(\eta u)=0, \\
\frac{\partial u}{\partial t}+u \frac{\partial u}{\partial x}+g \frac{\partial \eta}{\partial x}+\frac{1}{3} h_{0} \frac{\partial^{3} \eta}{\partial x \partial t^{2}}=0,
\end{array}
$$

which is obtainable from (3-4) by recognising that the dominant mechanisms are expressed in the leading approximation (32) and this shows the above transformation to be asymptotically correct. The dispersion relation of the new system (33-34) is

$$
\omega= \pm \sqrt{g h_{0}} k / \sqrt{1+\frac{1}{3} h_{0}^{2} k^{2}}
$$

which was wavelike behaviour for all wavenumbers, albeit it inaccurate for large wavenumbers. ${ }^{8}$ Furthermore, equations (33-34) are of lower-order in spatial derivatives and need only two boundary conditions at the extremes of the physical domain. These two boundary conditions will come from the consideration of the slow manifold in the original dynamics as outlined in Section 3.1.

The Korteweg-deVries equation for uni-directional long waves, equation (5), has a similar problem in that the spatial evolution of the model has eigenvalues $\lambda=0$, corresponding to the long waves being modelled, and the pure imaginary $\lambda= \pm i \sqrt{c_{0} / \gamma}$, which correspond to unphysical modes. Once again the arguments developed in Section 3.2 to provide boundary conditions for this model will not apply. The corresponding dispersion relation for linearised waves is

$$
\omega=k\left(c_{0}-\gamma k^{2}\right)
$$

which, while not unstable for high wavenumbers, has finite wavenumber waves, $|k|>\sqrt{c_{0} / \gamma}$, travelling in the wrong direction. Despite the many

\footnotetext{
${ }^{8}$ Similar considerations in beam theory lead to the mixed derivatives in Rayleigh's equation (6) and in Love's equation (7).
} 
fascinating exact solutions of (5), Benjamin et al. [2] have maintained that for modelling purposes it is desirable to modify the equation to the asymptotically equivalent

$$
\frac{\partial \eta}{\partial t}+c_{0}\left(1+\frac{3}{2 h_{0}} \eta\right) \frac{\partial \eta}{\partial x}-\frac{\gamma}{c_{0}} \frac{\partial^{3} \eta}{\partial x^{2} \partial t}=0
$$

In this form the model has spatial eigenvalues $\lambda=0$, corresponding to the long waves, and $\lambda \sim c_{0}^{2} /(\gamma p)$, corresponding to an unphysical mode. The arguments of Section 3.2 may then apply and this unphysical mode could be eliminated by imposing the correct boundary condition.

The relevant characteristics of the above two examples are typical. Consider a $\kappa$ th order truncated linearised version of the general model evolution equation (27). The spatial evolution away from the ends is obtained by substituting $\mathrm{s}=\mathrm{v} \exp (p t+\lambda x)$ to obtain the eigen-problem

$$
p \mathbf{v}=\mathscr{G}(\lambda) \mathbf{v}=\left(\mathscr{G}_{0}+\lambda \mathscr{G}_{1}+\lambda^{2} \mathscr{G}_{2}+\cdots+\lambda^{\kappa} \mathscr{G}_{\kappa}\right) \mathbf{v}
$$

for eigenvalues $\lambda$ and eigenvectors $\mathbf{v}$. The slow manifold approximation is based on the extreme slow evolution obtained by taking $p=0$. Thus the modes in the spatial evolution are determined by the characteristic equation $\operatorname{det}[\mathscr{G}(\lambda)]=0$. The assumption in Section 3.2 is that this has roots which are either zero (corresponding to the slow manifold) or have strictly non-zero real part; problems arise if there exist pure imaginary roots-the arguments of Section 3.2 will not work if it has roots $\lambda= \pm i \omega$. However, in this case the left-hand side of the characteristic equation may be factored to $\operatorname{det}[\mathscr{G}(\lambda)]=$ $\left(\lambda^{2}+\omega^{2}\right) f(\lambda)$. Then, when we consider the temporal evolution of the model (27), by trying solutions proportional to $\exp (\sigma t+i k x)$ and obtaining the characteristic equation $\operatorname{det}[\mathscr{G}(i k)-\sigma I]=0$ for the growth-rate $\sigma$ as a function of wavenumber $k$, it is apparent that $\sigma=0$ at wavenumber $k=$ $\pm \omega$. Thus the growth-rate, $\sigma(k)$, must change sign at the finite wavenumber $\omega$; consequently the high wavenumber behaviour of the model is opposite to the low wavenumber behaviour for which the model is derived. Hence the model is at least ill-adapted to the original problem, as in the KortewegdeVries equation (5), and may be intractably unstable, as in the Boussinesq equations (3-4). In such cases the model should be changed to one with better temporal behaviour, and simultaneously the difficulty in obtaining the appropriate boundary conditions will be overcome; that this is reasonably common is evidenced by other equations in the Introduction which have mixed derivatives.

What if the spatial evolution eigenproblem (36) has roots which are nearly pure imaginary, say $\lambda=\theta \pm i \omega$ for some small $\theta$ ? By itself this would similarly produce an undesirable change in the temporal growth-rate $\sigma$. 
However, many problems have reflectional symmetry in the $x$ direction and such a pair of eigenvalues could not occur in isolation; the pair must occur in a quadruplet $\lambda= \pm \theta \pm i \omega$. In this case the characteristic equation may be factored to

$$
\operatorname{det}[\mathscr{G}(\lambda)]=\left(\lambda^{2}-2 \theta \lambda+\theta^{2}+\omega^{2}\right)\left(\lambda^{2}+2 \theta \lambda+\theta^{2}+\omega^{2}\right) f(\lambda)=0,
$$

whence the temporal growth-rate near the critical wavenumber of $k= \pm \omega$ is

$$
\sigma \approx \frac{f(i k)}{g(i k)}\left[\left(\theta^{2}+\omega^{2}-k^{2}\right)^{2}+4 \theta^{2} k^{2}\right],
$$

where $g(\lambda)$ is related to the trace of $\mathscr{G}(\lambda)$. Since the expression in square brackets is never zero as $k$ is varied, the growth-rate does not change sign, the model is reasonably well behaved, and the arguments in Section 3.2 provide the model with appropriate boundary conditions.

Another model equation which is based on the slowly-varying approximation is the Kuramoto-Sivashinsky equation $(2)[15,31]$. Linearly, its temporal evolution is determined by growth-rates $\sigma=k^{2}\left(1-k^{2} / \Lambda^{2}\right)$ which displays instability for low wavenumbers; it is the presence of the nonlinearity transforming "energy" to the strongly dissipative high wavenumbers which permits bounded solutions of relevance to the original physical systems from which it is derived. This equation apparently displays the problematical change in sign of the growth-rate which accompanies pure imaginary modes in the spatial evolution. Indeed this is the case, the eigenvalues of the $p=0$ spatial evolutions are $\lambda=0$ (twice) and $\lambda= \pm i \Lambda$; consequently the arguments in Section 3.2 cannot be applied to give boundary conditions. In this sort of circumstance, the correct procedure is to realise that all four of these spatial modes must form the basis of the dynamical model. This may be achieved in the derivation of the Kuramoto-Sivashinsky equation (2) either by imagining $\Lambda$ is "small" ( $1 / \Lambda$ is to be large when compared with the ignored dynamical length-scales) in which case the model is a slow manifold model, or by forming an invariant manifold model, as mentioned in the previous sub-section, which is based on these four modes. In either case the appropriate four boundary conditions for the fourth-order Kuramoto-Sivashinsky equation will be obtained by the considerations of the original dynamics, as discussed in Section 3.1; it is only when the Kuramoto-Sivashinsky equation is modified by including terms of higher-order than four that the arguments in Section 3.2 would be needed.

\section{Conclusion}

Given the prevalence of continuum models based on the slowly-varying approximation, the issue of finding the appropriate boundary conditions is 
important but frequently overlooked. The centre manifold approach to deriving rational models provides a technique to obtain àsymptotically correct boundary conditions; other techniques of approximation generally cannot do this.

Here I have concentrated exclusively on models with one spatial dimension. The foundation of the approach is to investigate the evolution in the boundary layers near either end of the physical domain of interest. This is a fairly complicated procedure, but other work that I have done in the last few years suggests that, in general, these complications are unavoidable.

The outstanding challenge now is to derive rationally boundary conditions for mathematical models which are set in two or three spatial dimensions; for example, the evolution of a convective planform [28], and the Navier-Stokes equation, respectively.

\section{Acknowledgement}

This research was generously supported by a grant from the University of Adelaide. I would like to thank Prof. Ron Smith for posing the question in the first place.

\section{References}

[1] D. Armbruster, J. Guckenheimer \& P. Holmes, "Kuramoto-Sivashinsky dynamics on the center-unstable manifold" SIAM J. Appl. Math. 49 (1989) 676-691.

[2] T. B. Benjamin, J. L. Bona \& J. J. Mahoney, "Model equations for long waves in nonlinear dispersive systems" Phil. Trans. Roy. Soc. A 272 (1972) 47-782.

[3] J. Carr, “Applications of centre manifold theory" Applied Math. Sci. 35 (1981).

[4] C. J. Chapman \& M. R. E. Proctor, "Nonlinear Rayleigh-Bénard convection between poorly conducting boundaries" J. Fluid Mech. 101 (1980) 759-78.

[5] P. C. Chatwin, "The approach to normality for the concentration distribution of a solvent flowing along a straight pipe" J. Fluid Mech. 43 (1970) 321-352.

[6] P. H. Coullet \& E. A Spiegel, “Amplitude equations for systems with competing instabilities" SIAM J. Appl. Math. 43 (1983) 776-821.

[7] S. M. Cox \& A. J. Roberts, "Centre manifolds of forced dynamical systems" J. Austral. Math. Soc. B 32 (1991) 401-436.

[8] M. C. Cross, P. G. Daniels, P. C. Hohenberg \& E. D. Siggia, "Phase-winding solutions in a finite container above the convective threshold" J. Fluid Mech. 127 (1983) 155-183.

[9] K. B. Dysthe, "Note on a modification to the nonlinear Schrödinger equation for application to deep water waves" Proc. Roy. Soc. Lond. A 369 (1979) 105-114.

[10] J.-P. Eckmann \& C. E. Wayne, "Propagating fronts and the center manifold theorem" preprint (1990).

[11] Y. C. Fung, Foundations of solid mechanics, (Prentice-Hall, 1965). 
[12] J. Guckenheimer \& P. Holmes, Nonlinear oscillators, dynamical systems and bifurcations of vector fields, Springer-Verlag (1983).

[13] J. M. Hyman, B. Nicolaenko \& S. Zaleski, "Order and complexity in the KuramotoSivashinsky model of weakly turbulent interfaces" Physica D 23 (1983) 265-292.

[14] P. A. E. M. Janssen, "On a fourth-order envelope equation for deep-water waves" $J$. Fluid Mech. 126 (1983) 1-11.

[15] Y. Kuramoto, "Diffusion induced chaos in reactions systems" Progr. Theoret. Phys. Suppl. 64 (1978) 346-367.

[16] G. N. Mercer \& A. J. Roberts, "The application of center manifold theory to the dispersion of contaminant in channels with varying flow properties" SIAM J. Appl. Math. 50 (1990) 1547-1565.

[17] A. Mielke, "Saint-Venant's problem and semi-inverse solutions in nonlinear elasticity" Arch. Rat. Mech. \& Anal. 102 (1988) 205-229.

[18] _ , "On Saint-Venant's problem and Saint-Venant's principle in nonlinear elasticity" Trends in Appl. Maths. to Mech. (1988) 252-260.

[19] _ , "On Saint-Venant's problem for an elastic strip" Proc. Roy. Soc. Edin. A 110 (1988) 161-181.

[20] R. G. Muncaster, "Invariant manifolds in mechanics I: The general construction of coarse theories from fine theories" Arch. Rat. Mech. 84 (1983) 353-373.

[21] A. C. Newell \& J. Whitehead, "Finite bandwidth, finite amplitude convection" J. Fluid Mech. 38 (1969) 279-303.

[22] D. I. Oats \& A. J. Roberts, "The performance of invariant manifolds in modelling the dynamics of the Kuramoto-Sivashinsky equation" preprint (1991).

[23] A. J. Roberts, "Simple examples of the derivation of amplitude equations for systems of equations possessing bifurcations" J. Austral. Math. Soc. Ser. B 27 (1985) 48-65.

[24] _ "The application of centre manifold theory to the evolution of systems which vary slowly in space” J. Austral. Math. Soc. Ser. B 29 (1988) 480-500.

[25] __ "Appropriate initial conditions for asymptotic descriptions of the long term evolution of dynamical systems" J. Austral. Math. Soc. Ser. B 31 (1989) 48-75.

[26] A. J. Roberts, "The utility of an invariant manifold description of the evolution of dynamical systems" SIAM J. of Math. Anal. 20 (1989) 1447-1458.

[27] A. J. Roberts, "The invariant manifold of beam theory. Part I: the simple circular rod" preprint (1990).

[28] A. J. Roberts, "Planform evolution in convection-an embedded centre manifold" $J$. Austral. Math. Soc. Ser. B (1990) to appear.

[29] L. A. Segel, "Distant side-walls cause slow amplitude modulation of cellular convection" J. Fluid Mech. 38 (1969) 203.

[30] J. Sijbrand, "Properties of center manifolds" Trans. Amer. Math. Soc. 289 (1985) 431469.

[31] G. I. Sivashinsky, "On cellular instability in the solidification of a dilute binary alloy" Physica $D 8$ (1983) 243-248.

[32] R. Smith, "Diffusion in shear flows made easy: the Taylor limit" J. Fluid Mech. 175 (1987) 201-214.

[33] R. Smith, "Entry and exit conditions for flow reactors" IMA J. of Appl. Maths. 41 (1988) $1-40$.

[34] G. I. Taylor, "Dispersion of soluble matter in a solvent flowing through a tube" Proc. Roy. Soc. Lond. A 219 (1953) 186-203.

[35] R. Temam, "Inertial manifolds" Math. Intelligencer 12 (1990) 68-74.

[36] G. B. Whitham, Linear and nonlinear waves Wiley-Interscience (1974). 